\title{
Serge Valletti ou les jeux de l'abondance digressive
}

\author{
Jean-Pierre Ryngaert
}

\section{OpenEdition}

Journals

Édition électronique

URL : http://journals.openedition.org/recherchestravaux/149

DOI : 10.4000/recherchestravaux.149

ISSN : 1969-6434

\section{Éditeur}

UGA Éditions/Université Grenoble Alpes

\section{Édition imprimée}

Date de publication : 15 octobre 2006

Pagination : $9-15$

ISBN : 978-2-84310-099-4

ISSN : 0151-1874

\section{Référence électronique}

Jean-Pierre Ryngaert, « Serge Valletti ou les jeux de l'abondance digressive », Recherches \& Travaux [En ligne], 69 | 2006, mis en ligne le 25 novembre 2008, consulté le 08 septembre 2020. URL : http:// journals.openedition.org/recherchestravaux/149; DOI : https://doi.org/10.4000/recherchestravaux 149 


\section{Serge Valletti ou les jeux de l'abondance digressive}

Serge Valletti bénéficie d'un statut particulier dans notre paysage d'auteurs. On pourrait le caractériser par l'abondance et par une forme de générosité: celle de ses textes, celle de sa langue, celle des histoires qu'il raconte. Serge est un amateur de digressions, de volutes, d'explications copieuses entre personnages, de précisions infinies dont l'intérêt ne se mesure pas sur le moment, et parfois jamais; de tortueux mensonges développés entre spécialistes de la mythomanie et de calembours et d'à-peu-près à faire frémir. Ceci ne va pas sans risques. Acteur, Valletti excelle dans l'art difficile de se prendre les pieds dans le tapis, de rater la marche la plus facile, de déraper quand l'essentiel est derrière lui, accompli, et qu'il semble que plus rien ne peut lui arriver. C'est alors qu'il ne trouve plus la sortie, coincé qu'il est entre des pendrillons qui se sont ligués contre lui, et que tout tourne à la catastrophe.

Face à ce funambulisme du jeu dont on ne sait plus ce qu'il doit au vrai savoir-faire et à la fausse maladresse, à ces subtils ratages d'acteur roué mettant toute sa naïveté au service de l'interprétation, on peut chercher un équivalent dramaturgique, un goût pour le risque et l'errance transformée en art de la digression, une forme fondatrice de son théâtre. Ceci explique peut-être une certaine méfiance des milieux universitaires réputés sérieux pour un auteur qui écrit dans sa tête comme ça lui vient, qui a joué longtemps en duo et en solo, comme un enfant qui s'amuserait à écrire des récits monstrueux et qui s'étonnerait qu'on le prenne pour un écrivain.

Il garde d'ailleurs pour l'Université une ironie (pour le moins) d'ancien cancre qui a réussi à écrire en ayant raté les trois séries du baccalauréat. Déjà une digression! Sa posture favorite est de faire mine de ne pas faire grandchose, et de remplir le sac à billets comme en s'amusant, tel ce jour mythique où lui et ses copains adolescents avaient bourré un théâtre avec un spectacle 
qui était peut-être un canular et peut-être pas, et vendu tant de billets qu'il en rit encore aujourd'hui'.

On pourrait penser qu'un paresseux est celui qui choisit la ligne droite, la plus courte pour aller d'un point à un autre. Soit Valletti n'est pas paresseux, soit, comme le Dieu de Claudel, il préfere définitivement les lignes courbes et a adopté les chemins de traverse comme seul système géographique. Ceci ne va pas sans malentendus lorsque, comme je l'ai fait, en m'appuyant sur une trentaine d'années d'amitié, je lui ai déclaré qu'il «écrivait trop ", entendant sans doute par là qu'il fallait qu'il se relise un peu plus, ou qu'il accepte moins de commandes, ou qu'il supprime de temps en temps une digression plus obscure que les autres. Erreur dont je me repens, même s'il n'y avait là aucun désir de leçon. Copieux, courbe, abondant, généreux, voilà comme il écrit, et, même si là aussi il enjolive un peu le tableau, il écrit d'autant plus qu'on lui en demande plus, faisant du hasard et de l'accident les éléments qui président à son désir d'écrire. Davantage, comme certains plasticiens, amoureux du présent et ne faisant pas de différence entre leur pratique artistique et leur vie, que comme la plupart des dramaturges. Ainsi Valletti répète-t-il "dans sa tête» en traversant Paris (je le rencontre marchant rue de Rivoli; "Qu'est-ce que tu fais? - Je répète»); ainsi, faute de théâtre, aménage-t-il la cave du magasin de son amie pour en faire une salle pour deux spectateurs, et, précise-t-il, les gens achetaient des trucs au magasin en sortant ${ }^{2}$.

Parce qu'il ne faut pas se prendre au sérieux, que la posture de l'écrivain tourmenté est déjà prise, qu'il ne veut pas «se la jouer» ou alors qu'il se la joue réaliste (“j'écris pour manger», dit-il - et il aime manger), et pas trop occupé (le bonheur d'écrire c'est quand il a fini) et qu'il va pouvoir se reposer.

On peut donc entrer dans cette mythologie, y collaborer comme je le fais, et cependant, pousser un peu cette analyse des digressions et voir comment le jeu des voix s'organise dans des architectures toujours complexes qui n'en finissent pas d'ouvrir des parenthèses. Voilà donc une proposition en forme d'esquisse qui pourrait servir à l'étude systématique du développement de la parole dans les textes de Serge Valletti. On n'y verra ni système ni grille, mais un clin d'œil de l'universitaire à l'auteur: même le discours en apparence le plus désorganisé suit ici quelques principes récurrents, quand bien même ils seraient vraiment ou faussement involontaires.

I. Cf. Serge Valletti, «Itinéraire d'auteur», Centre national des écritures du spectacle, La Chartreuse de Villeneuve-lès-Avignon, 1999.

2. Ibid. et souvenir personnel de spectateur. 


\section{Lois de l'abondance digressive:}

I) Tout mérite de longues généreuses explications.

2) Pourtant, rien de ce qui est parfaitement connu des personnages, ne mérite des explications utiles au tiers lecteur ou spectateur.

3) Tout nouveau sujet doit être bien accueilli et volontiers développé.

4) Il est possible de changer de sujet aussi souvent que le nécessite la pensée mouvante de celui qui parle.

5) Tout sujet technique mérite des explications encore plus détaillées.

6) N'importe quel sujet peut devenir technique si l'on prend le temps d'en parler.

7) Tout parleur peut convoquer dans son discours autant d'interlocuteurs imaginaires qu'il en éprouve le désir : ceux-ci ne risquent pas de le contredire. Corollaire: il peut évoquer et nommer autant de personnages qu'il le veut, même et surtout s'ils demeurent inconnus du lecteur.

8) À force de paroles, il se dit toujours quelque chose. Il est donc également utile de parler "pour dire» que "pour ne rien dire».

I. Puisque rien n'est simple, tout peut devenir très compliqué dès lors qu'il faut expliciter le fonctionnement du monde dans ses moindres détails. Lénoncé élémentaire: «et voilà pas qu'un client qui sortait de la quincaillerie Jasper ${ }^{3}$ » réclame une série de précisions: où se trouvait la quincaillerie Jasper? (réponse: dans la rue, à côté du barbier). Le type entrait ou sortait? (réponse: il sortait probablement, sauf s'il rentrait à reculons). Appel à l'expérience qui confirme l'affirmation: celui qui parle a déjà vu un tel phénomène, attesté par les informations télévisées et par sa mère. Celle-ci regarde les informations, du moins quand il y a quelque chose d'intéressant à voir. Le phénomène d'explication peut ne jamais s'arrêter dès lors qu'il s'agit de faire partager son expérience à des auditeurs qui n'ont pas forcément la même, ou alors à qui il faut rafraîchir la mémoire. Chaque point mérite qu'on s'y arrête, chaque précision en entraîne de nouvelles (comment sa mère mesure-t-elle l'intérêt des informations quand elle ne les regarde pas?). Le monde des personnages de Valletti est si complexe que tout processus d'explication réclame temps, patience et réflexion!

2. Paradoxalement, Valletti fait pourtant mine d'ignorer les principes de l'information, parfois au moment où il en multiplie les détails. On sait comment, pour les classiques et les partisans du modèle dramatique qui s'imposa par la suite, celle-ci doit être claire et complète. La fameuse «double énonciation" de la communication théâtrale, souvent mise à mal ces dernières années, suppose que le tiers lecteur ou spectateur soit pris en compte par

3. Saint Elvis, Paris, Christian Bourgois, 1990, p. Iо. 
les discours des personnages. Dans son écriture, Valletti dérègle ce système ou fait mine de l'ignorer. Les échanges sont placés sous le signe de l'excès d'implicite ou de l'excès d'explicitation. Dans les deux cas, le tiers reste pantois devant un dialogue dont il semble exclu. Très implicite, l'échange se déroule à coups de précisions qui ne relèvent ni du domaine de la fable ni de celle de l'action. Tout se passe alors comme si les personnages ne prenaient jamais en compte l'éventuel témoin, et que, entièrement plongés dans leurs systèmes de référence, ils excluaient les éclaircissements "utiles». Ainsi les références propres aux personnages deviennent-elles les seules recevables dans l'univers qui se construit, qu'il soit question de sacs d'aspirateur ou de ramassage de coquillages, ou d'allusions à des personnes connues des seuls personnages et qui n'interviendront jamais dans le texte autrement que par ouï-dire.

3 et 4 . Ce que les linguistes, spécialistes de la conversation, appellent la coopération, est couramment pratiqué par les personnages de Valletti. Tout nouveau sujet de conversation rencontre généralement la plus grande disponibilité des "entreparleurs», qui relancent, interrogent ou contestent, mais en tout cas coopèrent, quel que soit le sujet. La sinuosité des discours est encore augmentée par la propension de tous les personnages à changer de sujet très souvent, et à revenir tardivement sur les sujets qui semblaient abandonnés. Plus le nombre de personnages est élevé, plus les sujets abordés sont nombreux et relancés par la coopération des intervenants, plus l'impression de sinuosité et d'abondance augmente. Quand les sujets concernent directement ou non une action entreprise (Carton plein) le comique vient du «frottement» entre tous ces sujets et l'action concrète en cours, plutôt simple. Le personnel dramatique de Valletti est toujours prêt à parler et à coopérer sinon pour faire "avancer " la parole, au moins pour développer ce qui est mentionné, aussi mince que soit la proposition conversationnelle.

5 et 6. Les interventions "techniques" figurent parmi les favorites des entreparleurs qui entament tous très volontiers un discours ou un débat autour d'un point précis et litigieux, qu'il s'agisse de la qualité du vin ou du système de contrôle propre aux bistrotiers (Pœub). Mais le spécialiste doit prendre son temps, et plus il est spécialiste, plus il est indispensable qu'il entre dans les détails, fussent-ils apparemment dénués d'intérêt pour le reste du monde. Si le spécialiste n'est pas un vrai spécialiste (ce qui est fréquent), rien ne l'empêche d'en prendre l'apparence, et en premier lieu le langage. Ainsi, à une demande de «brochette» d'un innocent client, Meredick s'improvise vendeur avisé et soucieux d'hygiène ${ }^{4}$ :

4. Le jour se lève, Léopold!, Paris, Christian Bourgois, I988, p. 50. 
Ah! non, mais alors! Vous avez vu le temps qu'il fait? [...] Qu'il pleut sans interruption depuis dix jours, que les camions sont bloqués Dieu sait où à cause des transformateurs qu'ils posent dans les routes qui viennent ici, que quand il pleut, les moules et les autres, les clams, ça sent, elles sentent, bien qu'elles soient dans l'eau, raison de plus! Qu'on les sort de là elles mettent un temps à crever. Vous vous rendez pas compte de ce que vous dites sans savoir. Y a pas de sot métier, mais y a des fabricants d'andouilles, ça revient kif-kif au même. "Je ne me rends pas compte de ce que je dis et je veux des brochettes par un temps où ça coule comme des pendulettes." Sans savoir. [...] vous risquez de passer sans vous en rendre compte mon pauvre ami...! Le Pauvre, peuchère...!

Comme quoi le discours technique peut mettre en garde un amateur de brochettes qui s'expose ingénument à la mort.

Quand deux discours techniques s'opposent, Valletti réalimente des conflits d'autant plus violents qu'ils n'ont pas de cause apparente ou bien entraîne ses personnages dans une discussion privée, obscure pour les non-spécialistes, autour d'une marque de sacs d'aspirateurs (Le jour se lève, Léopold!) ou le découpage du saucisson par un charcutier (Carton plein).

7. Dans les solos, le personnage garde évidemment le contrôle absolu sur les créatures qu'il convoque et qu'il fait parler à sa guise, selon un principe bien éprouvé dans le one man show où le monologue autorise l'ouverture de dialogues éphémères avec des silhouettes rapidement convoquées ou avec des spectateurs qu'il s'agit (faussement) de «faire parler». Bien entendu, il ne reste qu'un seul parleur qui mène le jeu et se garantit la toute-puissance. Même dans ses textes où interviennent plusieurs personnages, Valletti fait convoquer par celui qui parle d'autres parleurs potentiels, parfois emboîtés les uns dans les autres - leur texte demeurant suspendu. C'est le cas par exemple dans Saint Elvis où, d'entrée, Elvis convoque "un client qui sortait de la quincaillerie Jasper", qu'il appelle "le type», et sa mère Gladys, ce qui retarde l'intervention du "type». Et finalement, il choisit de s'adresser à un interlocuteur du public ("mon frère», puis "nous sommes tous frères" en reprise de "eh ben mon vieux»). L'accumulation des voix et des adresses qui se croisent emplissent tout l'espace de la parole et le personnel de la fiction augmente au gré des besoins du parleur principal, qui a toujours raison ( La vérité, c'est ce que je dis, et c'est pas rien d'autre, tu crois pas, mon frère?»). La multiplication de parleurs fictifs, à l'intérieur d'un texte déjà fertile en digressions, crée l'impression d'une foule toujours prête à prendre la parole, à donner son avis, à entraîner le discours là où elle veut qu'il aille. Il se crée donc un espace de parole d'autant plus ouvert qu'il est apparemment libre d'accès et que le nouveau parleur n'a besoin d'aucune "ratification ", au sens des linguistes, pour entrer dans la conversation. Faux personnages dans les 
solos, ceux-ci peuvent se matérialiser dans un texte comme Pœub où plus d'une vingtaine de figures prennent la parole.

8. La question du sens ne dépend évidemment pas des énoncés. Ce qui se dit chez Valletti a toujours l'air de se construire sur le vide, ou contre le silence, comme si c'était bien la panne définitive qui était le plus gros risque encouru par les personnages. L'accueil forcené de «tout ce qui peut se dire» dans le plus grand désordre apparent fait du détour ou de la digression la forme la plus évidente des textes. Pourtant, personne ne parle jamais vraiment "pour ne rien dire». L'accordage des voix, l'être ensemble, le départ de l'aventure verbale la plus folle ou la plus facétieuse, sont des pistes de travail, au même titre que la résistance à l'esprit de sérieux ou le besoin de parler «à côté». Jamais dans le sens de la marche, toujours là où l'on ne l'attend pas, la plus folle conversation rend compte à sa façon de l'inépuisable opacité du monde.

Puisque les textes de Valletti avancent toujours à coups de digressions, on peut se demander, habitués que nous sommes aux structures classiques, où en est le centre, et si ça finit par avancer. Parfois, il s'agit quasiment de débuts successifs, d'autant d'appendices narratifs qui semblent se caractériser par leur gratuité. Ce qui est de nature à provoquer l'agacement de certains spectateurs qui cherchent une progression logique, mais la jubilation de ceux qui partagent le goût de l'auteur pour la réinvention perpétuelle. Dans ce qui ressemble à une stratégie d'évitement, l'essentiel n'est plus repérable, ou plutôt, s'il n'est pas au centre, on le chercherait en vain ailleurs que dans cette vaporisation de l'imaginaire, comme si le cœur des choses gisait dans les détails. Le personnage ne parle pas comme il devrait parler, les événements éventuels sont toujours retardés, la parole toute-puissante est le moteur d'infatigables bavards qui, précisément, ne semblent jamais être en mesure d'aller à l'essentiel. L'essentiel existe-t-il d'ailleurs autrement que dans cette urgence du présent qui fait que ce qui doit être dit est dit à ce moment-là, littéralement toutes affaires cessantes? Les questions doivent être traitées les unes après les autres, en n'étant jamais soumises à une grande action ni à un grand projet, mais en fonction du moment. La dictature du présent, ou le goût de l'instant, ont à voir avec l'enfance et le plaisir de raconter des histoires ou de forger des mensonges. Valletti, mythomane professionnel, se livre avec délices au plaisir de l'instant, il ne résiste ni à une histoire nouvelle, ni à l'évocation d'un autre personnage, ni évidemment à un bon mot. Ce qui provoque toujours, on le sait bien, des embrouilles supplémentaires, qui participent aux sinuosités récurrentes des intrigues.

Valletti a été l'un des premiers dramaturges de sa génération à être saisi par la toute-puissance de la parole. Pendant longtemps, il a été, hors scène ou sur 
scène, l'initiateur de celle-ci, comme auteur et comme acteur. Le seul tireur de ficelles installé au bout du comptoir, en tête à tête avec son imaginaire. Même dans ses premiers duos, le partage de la parole s'arrangeait aisément, par un travail de bricolage et de renvois de balles. Les choses se sont sérieusement compliquées avec la multiplication des personnages et des voix dans Le jour se lève, Léopold! (son premier texte pour plusieurs personnages), chaque nouvelle voix apportant avec elle sa dose de complexité narrative. Car tous les personnages de Valletti ont en commun cette propension à la parole envahissante et contournée. Porteurs de la même caractéristique, ils multiplient d'autant l'embrouillage narratif général, l'auteur leur accordant également d'importance et le même droit à la digression. Digresseurs and co, ce personnel théâtral ne saurait se réduire à une explication simple et vaguement folklorique de Marseillais tchatcheurs. Ils parlent parce qu'ils ne peuvent pas faire autrement, ils parlent parce que c'est leur façon de construire ces univers emboîtés et baroques qui se ressemblent tous et sont leur signe d'appartenance à la tribu Valletti: les défieurs de silence. 